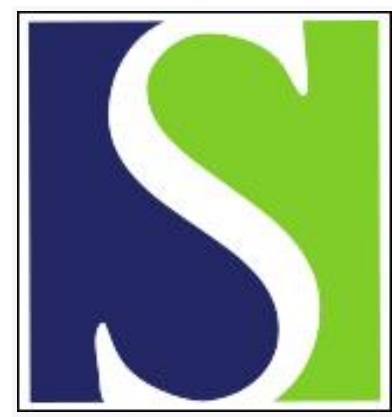

Scand J Work Environ Health 2012;38(5):467-475

https://doi.org/10.5271/sjweh.3260

Published online: 04 Nov 2011, Issue date: 01 Sep 2012

Effect of individualized worksite exercise training on aerobic capacity and muscle strength among construction workers - a randomized controlled intervention study

by Gram B, Holtermann A, Søgaard K, Sjøgaard G

Affiliation: Institute of Sports Sciences and Clinical Biomechanics. University of Southern Denmark, Campusvej 55 DK-5230 Odense M. bgram@health.sdu.dk

Refers to the following texts of the Journal: 2010;36(5):357-365 2007;33(6):405-424

The following articles refer to this text: 2013;39(5):456-467; 2015;41(2):140-152; 2016;42(4):291-298

Key terms: aerobic capacity; cardiovascular disease; cardiovascular health; construction worker; exercise; fitness; intervention; leisure-time physical activity; muscle strength; occupational health; occupational health; occupational physical activity; occupational setting; physical activity; physical fitness; physical heavy work demands; randomized controlled intervention; randomized controlled trial; worksite exercise

This article in PubMed: www.ncbi.nlm.nih.gov/pubmed/22057836

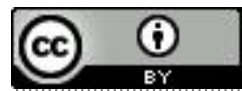




\title{
Effect of individualized worksite exercise training on aerobic capacity and muscle strength among construction workers - a randomized controlled intervention study
}

by Bibi Gram, MSc, ${ }^{1}$ Andreas Holtermann, PhD, ${ }^{2}$ Karen Søgaard, PhD, ${ }^{1}$ Gisela Sjøgaard, DrMedSc ${ }^{1}$

\begin{abstract}
Gram B, Holtermann A, Søgaard K, Sjøgaard G. Effect of individualized worksite exercise training on aerobic capacity and muscle strength among construction workers - a randomized controlled intervention study. Scand $J$ Work Environ Health. 2012;38(5):467-475. doi:10.5271/sjweh.3260
\end{abstract}

\begin{abstract}
Objectives The combination of high physical work demands and low physical capacity has been shown to increase the risk for cardiovascular disease and musculoskeletal disorders. The aim of this study was to assess the physical capacity of construction workers and evaluate the effect of individually tailored exercise programs on their physical fitness and muscular capacity.
\end{abstract}

Method The study was a randomized controlled trial of male constructions workers allocated to either an exercise or control group. The intervention lasted 12 weeks, and the exercise group trained $3 \times 20$ minutes a week. The participants completed health checks before and after the intervention period. Data from the first health check were used to tailor the exercise in the interventions.

Results At baseline, participants had maximal oxygen consumption $\left(\mathrm{VO}_{2 \max }\right)$ of 2.9 [standard deviation (SD) 0.7 ] $1 / \mathrm{min}$ and body mass index (BMI) of 28.3 (SD 4.7). Compared to representative data on employees in Denmark $(\mathrm{N}=78)$, this study population $(\mathrm{N}=67)$ had significantly lower relative aerobic capacity [difference in $z$-score -1.13 , standard error (SE) 0.1, $\mathrm{P}<0.001$ ] and higher BMI [difference in $z$-score $1.10, \mathrm{SE} 0.2, \mathrm{P}<0.001$ ] at baseline. With respect to the intervention, group $\times$ time analyses showed a significant difference in estimated change in $\mathrm{VO}_{2 \max }$ of $0.4 \mathrm{l} / \mathrm{min}$ for the exercise group and $0.0 \mathrm{l} / \mathrm{min}$ for the control group $(\mathrm{P}<0.001)$. Body mass and other general health measures remained unchanged.

Conclusion Training for 20 minutes, 3 times a week significantly increased $\mathrm{VO}_{2 \max }$ with a clinically relevant magnitude regarding risk of cardiometabolic disorders. This study demonstrates a good effectiveness for integrating short exercise bouts into organizational routines among constructions workers.

Key terms cardiovascular disease; cardiovascular health; fitness; leisure-time physical activity; occupational health; occupational physical activity; occupational setting; physical activity; physical fitness; physical heavy work demands; randomized controlled trial.

Physical activity during leisure time is well documented to improve cardiovascular and musculoskeletal health $(1,2)$. In contrast, high physical work demands have been shown to increase the risk for cardiovascular mortality among men with low physical fitness, but not among men with high physical fitness (3).

Therefore, workers with a mismatch between physical work demands and physical capacities (eg, physical fitness and muscular capacity) may particularly be at excessive risk for impared cardiovascular and musculoskeletal health (4).

Although leisure-time physical activity is known to improve physical capacity $(5,6)$ several studies have indicated that occupational physical activity does not $(3,6-7)$. Because high occupational physical activity increases the risk for being physically inactive during leisure time (8), workers with high physical work demands are at risk for having low physical capacity. Therefore, leisure-time physical activity may be of particular importance for decreasing the excessive risk for cardiovascular disease and musculoskeletal disorders among men with high physical work demands.

Construction workers have high physical work demands characterized by forceful muscle contractions,

1 Institute of Sports Science and Clinical Biomechanics, University of Southern Denmark, Odense, Denmark.

2 National Research Centre for the Working Environment, Copenhagen, Denmark.

Correspondence to: Bibi Gram, Institute of Sports Sciences and Clinical Biomechanics. University of Southern Denmark, Campusvej 55 DK-5230 Odense M. [E-mail: bgram@health.sdu.dk]. 
awkward postures, and unexpected physical loads (9). Accordingly, Dutch construction workers were shown to have $20 \%$ higher risk for cardiovascular disease compared to the general Dutch working population (10). Moreover, the prevalence of musculoskeletal disorders are high among construction workers and, together with cardiovascular diseases, have thus been found to be a major cause of disability among construction workers $(11,12)$.

Although the physical capacities of construction workers remain unknown, workplace health-promoting initiatives for improving physical capacity among construction workers may offer particularly high health benefits. However, controlled trials aiming to improve physical capacity among construction workers are lacking.

To improve the effectiveness of workplace healthpromoting interventions among workers with high physical work demands, it may be necessary to tailor the intervention to the individual characteristics of the worker so that the physical training matches the physical capacity of the individual worker.

Therefore, the aim of this study was to investigate the physical resources of construction workers and assess if an individually tailored exercise intervention would increase aerobic capacity or maximal oxygen consumption $\left(\mathrm{VO}_{2 \max }\right)$ and muscle strength among construction workers.

\section{Methods}

\section{Study design}

The study was a 12 -week randomized controlled trial with an exercise intervention and health check before and after. Participants were individually randomized into an exercise or control group. The randomization was balanced with respect to age and workplace and was performed blinded after the first health check. This study is a part of the FINALE (frame interventions for preserved work ability; long-term effect) program, a framework for health-promoting interventions in four Danish occupational groups, previously described as the FINALE construct (4).

Written informed consent was obtained from all participants before enrolment in the study. Results from the first health check were used to tailor individualized exercise programs. The exercise programs consisted of aerobic capacity and muscle strength training and are described in detail below.

The Region of Ethics Committee of Southern Denmark (No 20090058) approved the study protocol, and the study is registered in www.clinicaltrials.com (number NCT01007669).

\section{Subjects}

Study participants were employees working in the construction industry. They were recruited from three workplaces and companies in Denmark. The inclusion criterion was that the participants should have physically demanding tasks with high peak loads at work. The exclusion criterion was working $<20$ hours per week. There were 154 eligible participants when we stopped recruitment. In total, 67 participants accepted the invitation to join the study and were randomized to either the exercise $(\mathrm{N}=35)$ or control group $(\mathrm{N}=32)$ (see figure 1).

\section{Health check}

The health exam included measures of $\mathrm{VO}_{2 \max }$, isometric muscle strength, body mass, percent fat, blood pressure, and blood lipid profile. All participants were individually notified of the results shortly after the health check.

\section{Intervention}

The exercise program was performed during working hours, on or nearby the working place, and the training was implemented in cooperation with the employer. The program lasted 1 hour a week $(3 \times 20$ minutes $)$ for 12 weeks and was supervised by skilled instructors in two of the three weekly sessions.

The individual training programs were composed of aerobic and strength training. The exercise composition was tailored based on estimated $\mathrm{VO}_{2 \max }(13)$ and tests of maximal muscle strength in three body regions: neck-shoulder (two tests), abdomen-back (two tests) and hip-knee (one test) (14). Individual test results were compared to reference values from the Danish working population (14). If the test value was $<80 \%$ of reference value, the corresponding training element was included in the individual training instruction. Regarding strength training, the three body regions were considered separately, and training included only those regions that met the $80 \%$ criteria for $\geq 1$ tests. Participants with all their test values $>80 \%$ of reference value trained the capacity that was lowest on the job-group level. All training sessions included 10-minute dynamic exercises for warm-up and aerobic capacity (increasing from $\sim 50-70 \%$ estimated maximal workload) followed by 10 minutes of individually tailored exercises. Each participant received their own individual exercise protocol in a training diary that had to be completed at each training session. Exercises for aerobic capacity included bicycling and rowing, while exercises for strength training were selected from 11 different exercises [3 for neck-shoulder: (i) lateral raises, (ii) shrugs, and (iii) rows; 5 for abdomen-back: 


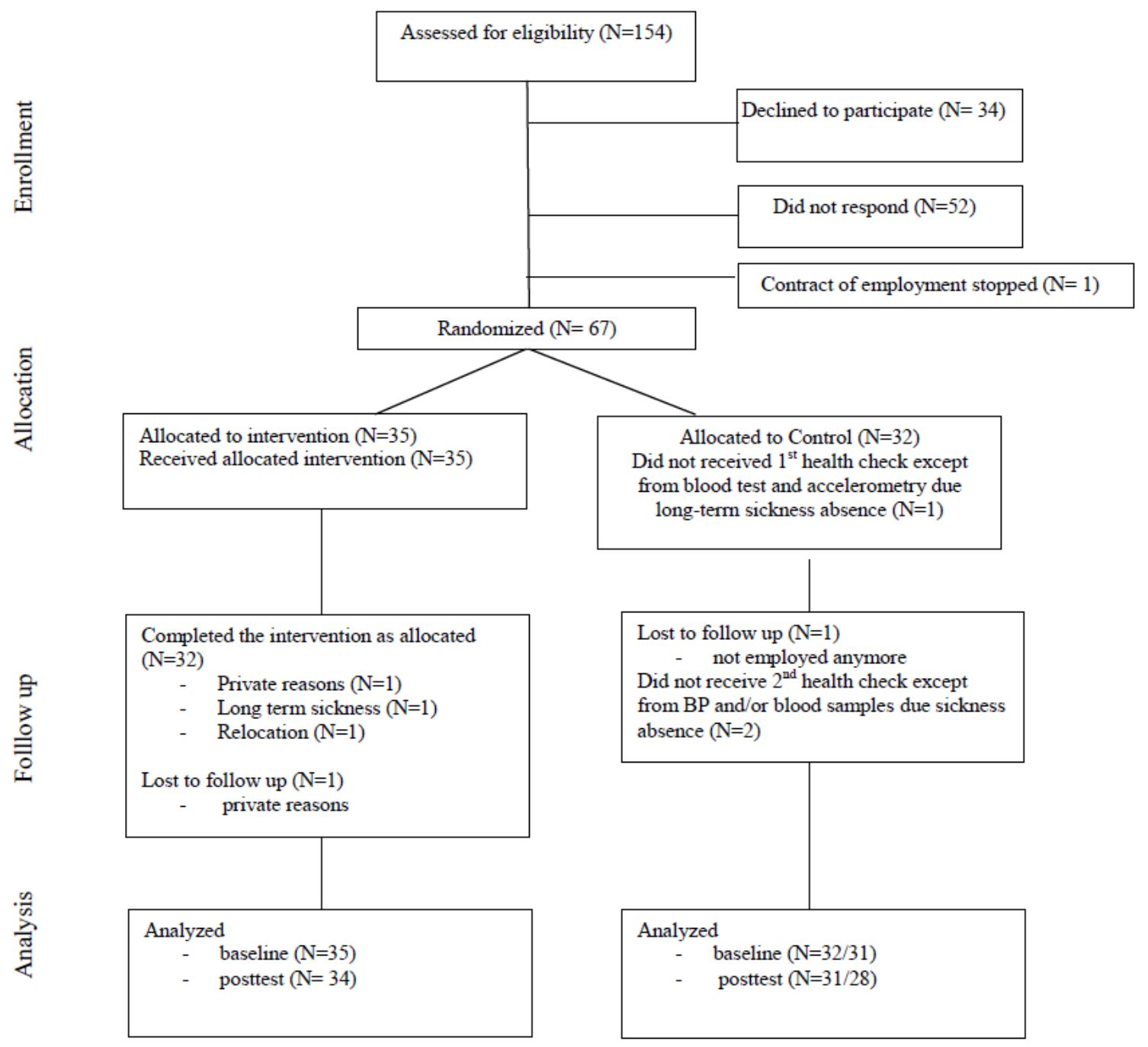

Figure 1. Flowchart of the trial. [BP=blood pressure.]

(i) back extensions, (ii) "bird dog", (iii) "the plank", (iv) crunches, and (v) oblique crunches; and 3 for hipknee: (i) static lunges, (ii) step-ups, and (iii) hip abductions] that could be graded by dumbbells/ resistance bands or body postures.

The intensity of the muscle strength training was approximately $60 \%$ of one repetition maximum (RM) and the intensity of the aerobic capacity training was at least $70 \%$ of $\mathrm{VO}_{2 \max }$. Two times during the 12-week training period, the participants were tested regarding the intensity of both aerobic capacity and muscle strength training. They were supervised at every session and, if necessary, intensity was adjusted for both muscle strength and - based on the Borg rated perceived exertion (RPE 6-20) scale - aerobic capacity training. Target RPE was 17.
The control group was not offered exercise training but rather a one hour lecture on general health promotion.

The exercise program and the health promotion lecture were the only intervention variables that differed between the groups. All participants were instructed to maintain their habitual lifestyle. We registered attendance rate to the exercise program and recorded absence cause.

\section{Outcome measures}

All measurements at baseline were performed before the randomization and repeated after the intervention. The primary outcome variables were $\mathrm{VO}_{2 \max }$ and isometric muscle strength. Secondary outcomes were body mass index (BMI), fat percent, blood pressure, and blood lipid profile. 
Maximal oxygen uptake $\left(\mathrm{VO}_{2 \max }\right)$

Maximal oxygen uptake was estimated from the relation between sub-maximal workload and stable heart rate obtained in Åstrand one-point sub-max test on a bicycle and subsequently using the Åstrand nomogram (13). $\mathrm{VO}_{2 \max }$ was corrected for age also according to Åstrand (15). The test procedure started with a load of 100 watts on a $1.0 \mathrm{~kg}, 80 \mathrm{rpm}$ Monark bicycle (Monark 874E, Monark Exercise AB, Sweden). During the first 2 minutes, the load was adjusted based on the measured heart rate. If the heart rate was $<120$ beats per minute (BPM) during the first 2 minutes, 0.5 $\mathrm{kg}$ was added; further weight was added at 3 and 4 minutes if needed to attain a stable heart rate between 120-170 BPM. When heart rate was recorded as stable for 1 minute, the test was terminated. The duration of each test was approximately 7-10 minutes. The post-intervention test was performed following the same protocol regarding pedal rate and weights added to the bicycle (ie, with the same external workload and duration at each incremental load as in the pre-randomization test). Heart rate was thus the only parameter that could differ between the prerandomization and post-intervention tests.

\section{Maximal voluntary contraction}

Isometric muscle strength was measured with a Bofors MODEL dynamometer (Bofors Elektronik, Karlskoga, Sweden) in five tests: (i) shoulder strength (dominant) shoulder elevation (dominant), (ii) arm abduction (dominant) abdominal strength, (iii) back strength, (iv) knee extension (right and left), and (v) handgrip (dominant). Three maximal voluntary contractions (MVC) with 30 seconds of recovery between attempts were conducted, and the highest value was recorded. Maximal force and the corresponding moment arm were registered (16). Furthermore, handgrip strength was measured with a handheld dynamometer (model 281111, Smedley, Tokyo, Japan).

\section{Blood pressure}

Supine blood pressure (BP) was measured after a 5-minute rest using a sphygmomanometer (Omron M7, OMRON Corporation, Kyoto, Japan). Three measurements were taken, and the mean of the two lowest measures was applied.

\section{Blood lipid profile}

Fasting blood samples was analyzed continually by standard methods (enzymatic colorimetric method).

\section{Statistical analysis}

The statistical analyses were based on an intention-to treat approach via STATA SE10 (StataCorp LP, College Station, TX, USA). Missing values in either baseline or post measurements were substituted with data carried forward and backward. When measurements had missing values in both baseline and post measurement these were replaced by means of all existing values within the particular variable. Differences between groups in $\mathrm{VO}_{2 \max }$, muscle strength, and anthropometry variables were tested using analyses of covariance (ANCOVA). Attendance based on records of training session participation was calculated as percentage of sessions attended of the 36 offered.

When analyzing effect of strength training on the three specific body regions in the respective strength training groups, the subjects from the control group were matched to the exercise group by using the same criteria of inclusion (ie, the controls were divided into the strength training groups they would have belonged to if they had been in the intervention group). Significant changes were estimated using one-way analysis of variances (ANOVA). In post-hoc analyses, paired t-tests were used to estimate significant differences within groups.

A representative group of employees in Denmark has been tested using the same procedure and methods as this study (14). To allow comparison between our study's random sample and the representative group, calculations of $z$-scores for relative $\mathrm{VO}_{2 \max }$, muscle strength (abdomen, back, dominant shoulder, dominant arm, dominant hand), and BMI were done with the following equation:

$z=$ (measured value - average value in the reference population $) /(\mathrm{SD}$ of reference population $)$

The means were stratified by age: $<30$ years, $30-40$ years and $>44$ years. To calculate $z$-scores for blood pressure, we used a sample of men in Denmark [from the Copenhagen Heart Study (17)] and the means were stratified by age: $20-29,30-39,40-49,50-59,60-69$ years. To calculate $z$-scores for lipid profile, we used as reference data a cohort from 2002, described as one of four population-based samples in a study from Sweden (18) and the means were stratified by age: $25-34,35-44$, 45-54 and 55-64 years.

Differences between these groups were calculated by unpaired two sample t-tests.

Sample size calculation was based on a minimal relevant difference in muscle strength of $10 \%$ and with a type 1 error of $5 \%$ and a power of $80 \%$ and showed a requirement of 46 participants in each group.

Data are shown as means and SD (table 1 and abso- 
lute values in table 2); group mean differences are shown as means and standard error (SE) and presented with $95 \%$ confidence intervals $(95 \% \mathrm{CI}) . z$-scores are shown as means and SE. Results were considered statistically significant if the 2-tailed $\mathrm{P}$-value was $<0.05$.

\section{Results}

Figure 1 shows the flow chart of the study. Two participants dropped out before follow up, two participants did not complete all tests in the health check due to sickness absence. The allocations to the individually tailored training are shown in figure 2. Six participants strength trained only one body region, 13 participants strength trained 2 body regions, and one participant trained all 3 body regions.

\section{Baseline}

At baseline there were no statistically significant differences between the groups in any of the variables (table 1).

Participants in this study had significantly higher BMI than a representative group of employees in Denmark with a mean difference in $z$-score 1.10 (SE 0.2, $\mathrm{P}<0.001)$. Furthermore they had significantly higher muscle strength in the abdomen (difference in $z$-score 0.76 , SE 0.2, $\mathrm{P}<0.001$ ), shoulder (difference in $z$-score 0.56 , SE $0.1, \mathrm{P}<0.001$ ), and arm (difference in $z$-score 0.29 , SE $0.1, \mathrm{P}<0.05$ ). In addition, this study population had significantly lower relative $\mathrm{VO}_{2 \max }$ (difference in $z$-score -1.13 , SE $0.1, \mathrm{P}<0.001$ ) than a representative group of employees in Denmark (14).

The participants in our study had significantly lower total cholesterol (difference in $z$-score -0.26 SE 0.1, $\mathrm{P}<0.05$ ) compared to the Swedish reference group while for BP, high- and low-density lipoproteins (HDL and LDL), and triglycerides there were no significant differences between the participants in this study compared to the Danish and Swedish references $(17,18)$.

\section{Intervention}

Adherence. The overall average attendance rate for the intervention group was $68 \%$. Of the participants, $59 \%$ had an attendance rate of $>70 \%$ and $75 \%$ of the participants had an attendance rate of $\geq 50 \%$.

The reason for an attendance rate of $<70 \%$ was either (i) absence due to illness $(\mathrm{N}=2)$ or vacation $(\mathrm{N}=2)$, (ii) pain in the musculoskeletal system $(\mathrm{N}=2)$, or (iii) lack of motivation $(\mathrm{N}=8)$. Eight participants (24\%) had an attendance rate of $\leq 50 \%$ due to lack of motivation.
Physiological outcomes. The absolute changes from preto post-intervention are shown in table 2. The exercise group had a significantly higher increase in $\mathrm{VO}_{2 \max }$ compared to the control group: $\Delta 0.401 / \mathrm{min}, \mathrm{P}=0.00$, while body weight did not change from pre- to postintervention in either group. Therefore, the $\mathrm{VO}_{2 \max } / \mathrm{kg}$ body weight increased significantly more in the exercise group [from 27.1 (SD 6.9) to 31.0 (SD 7.3) $\mathrm{ml} / \mathrm{min} / \mathrm{kg}$ ] compared with the control group [from 26.5 (SD 6.4) to 26.7 (SD 7.2) $\mathrm{ml} / \mathrm{min} / \mathrm{kg}$ ], $\mathrm{P}<0.05$.

The average watts used in the bicycle tests for both pre- and post-intervention tests were 130.3 (SD 24.4) in the intervention group and 126.3 (SD 25.1) in the control group.

In different age groups (20-39, 40-49, 50-63 years), at baseline there was an uneven distribution of relative $\mathrm{VO}_{2 \max }$ in the categories "very poor", "poor", "fair", "average", and "good" (22) The oldest had the relatively highest $\mathrm{VO}_{2 \max }$ for those $>50$ years, 4 out of 18 participants $(22 \%)$ at baseline had $\mathrm{VO}_{2 \max }$ corresponding to the "average" or "good" categories, while in the youngest group ( $<40$ year) no one reached the "average" category: only 4 of $21(19 \%)$ were placed in the "fair" category and the rest were placed in the "poor" and "very poor" category. In the age group 40-49 years, only 1 participant could be placed in the "average" category, 7 of 26 participants (27\%) had at baseline relative $\mathrm{VO}_{2 \max }$ corresponding to the "poor" category and 16 participants $(62 \%)$ were in the "very poor" category.

In the exercise group, the youngest men $(<30$ years old, $\mathrm{N}=4$ ) increased their relative $\mathrm{VO}_{2 \max }$ the most [from 32 (SD 8.4) to 38 (SD 5.2) 1/min], $\mathrm{P}<0.05$.

There were no significant changes in isometric maximal muscle strength; BMI; fat percentage; BP; total, HDL, or LDL cholesterol; or triglyceride levels (table 2).

\section{Post-hoc analysis}

Subsequent post-hoc analyses with focus on detailed issues were performed on intention-to-treat data.

Concerning analyses for each of the different strength training groups $(\mathrm{N}=20)$ and the corresponding control group $(\mathrm{N}=21)$, the between-group analyses showed that, for exercise versus control, there were no significant changes in muscle strength before or after the intervention neither when looking at the overall percentage change nor the body region-specific training groups, separately. Analyses within groups (paired t-test) did not show any changes regarding muscle strength. 
Table 1. Baseline demographic and clinical characteristics of study population. P values for the 1-way analysis of variances (ANOVA). [BP=blood pressure; HDL=high-density lipoprotein; LDL=low-density lipoprotein; SD=standard deviation; $95 \%$ Cl=95\% confidence interval.]

\begin{tabular}{|c|c|c|c|c|c|c|c|}
\hline \multirow[t]{2}{*}{ Characteristics } & \multicolumn{2}{|c|}{ Exercise group $\mathrm{N}=35$} & \multicolumn{2}{|c|}{ Control group $\mathrm{N}=32$} & \multicolumn{3}{|c|}{ Total (range) N=67 } \\
\hline & Mean & SD & Mean & SD & Mean & SD & $95 \% \mathrm{Cl}$ \\
\hline Age (years) & 44 & 11.1 & 43 & 10.0 & 43.7 & 10.5 & $21.9-63.4$ \\
\hline Weight $(\mathrm{kg})$ & 91.2 & 15.8 & 89.2 & 19.8 & 90.2 & 17.7 & $63.2-141.4$ \\
\hline Body mass index $\left(\mathrm{kg} / \mathrm{m}^{2}\right)$ & 28.8 & 4.1 & 27.9 & 5.2 & 28.3 & 4.7 & $18.9-44.6$ \\
\hline Fat $(\%)$ & 26.2 & 5.6 & 24.8 & 6.1 & 25.5 & 5.8 & $9.8-34.7$ \\
\hline $\mathrm{VO}_{2 \max }(\mathrm{I} / \mathrm{min})$ & 2.4 & 0.5 & 2.3 & 0.5 & 2.3 & 0.5 & 1.1-3.6 \\
\hline HR at steady state (bpm) & 148 & 16 & 150 & 14 & 149 & 2 & $149.3-153.9$ \\
\hline $\begin{array}{l}\text { Relative } \mathrm{VO}_{2 \max }(\mathrm{ml} / \mathrm{min} / \mathrm{kg}) \\
\text { Isometric muscle strength (nm) }\end{array}$ & 27.1 & 6.9 & 26.5 & 6.4 & 26.8 & 6.6 & $13.6-43.0$ \\
\hline Shoulder (dominant) & 139.5 & 40.3 & 140.8 & 34.6 & 140.1 & 37.0 & $65.6-265.1$ \\
\hline Arms (dominant) & 77.2 & 19.1 & 75.1 & 30.8 & 76.2 & 25.2 & 33.4-201.1 \\
\hline Abdomen & 220.3 & 52.6 & 219.4 & 56.2 & 219.9 & 54.4 & $128.4-380.3$ \\
\hline Back & 224.3 & 69.5 & 214.8 & 56.2 & 219.8 & 63.2 & 93.4-435.3 \\
\hline Leg (right) & 188.1 & 53.6 & 190.5 & 66.1 & 189.2 & 59.4 & $82.5-360.9$ \\
\hline Leg (left) & 176.4 & 49.3 & 186.1 & 61.9 & 181.0 & 55.5 & $91.6-359.9$ \\
\hline Handgrip (dominant) & 53.3 & 8.9 & 54.0 & 9.2 & 53.6 & 9.0 & $26.0-72.0$ \\
\hline Systolic BP (mm Hg) & 135 & 14 & 132 & 17 & 133.6 & 15.4 & $107-171$ \\
\hline Diastolic BP (mm Hg) & 86 & 10 & 85 & 11 & 85.2 & 10.5 & $65-115$ \\
\hline Total cholesterol (mmol/l) & 5.1 & 0.9 & 5.2 & 1.1 & 5.2 & 1.0 & $3.0-7.3$ \\
\hline HDL cholesterol (mmol/l) & 1.3 & 0.3 & 1.4 & 0.3 & 1.3 & 0.3 & $0.8-2.4$ \\
\hline LDL cholesterol (mmol/l) & 3.2 & 0.7 & 3.3 & 1.0 & 3.3 & 0.9 & $1.5-5.4$ \\
\hline Triglyceride (mmol/l) & 1.4 & 0.9 & 1.4 & 0.6 & 1.4 & 0.7 & $0.6-4.1$ \\
\hline
\end{tabular}

Table 2. Summary results of changes for each study group after 12 weeks intervention. Changes in post/pre-intervention values are absolute and not adjusted. Differences are estimated as the difference between means with $95 \%$ confidence intervals $(95 \% \mathrm{Cl})$, based on the 1-factor analysis of covariance (ANCOVA) with the level at baseline applied as a covariate. [BP=blood pressure; HDL=high-density lipoprotein; LDL=low-density lipoprotein; SD=standard deviation; SE=standard error.]

\begin{tabular}{|c|c|c|c|c|c|c|c|c|}
\hline \multirow[t]{2}{*}{ Characteristics } & \multicolumn{2}{|c|}{$\begin{array}{c}\text { Exercise }(\mathrm{N}=35) \\
\text { Post/pre intervention }\end{array}$} & \multicolumn{2}{|c|}{$\begin{array}{c}\text { Control }(\mathrm{N}=32) \\
\text { Post/pre intervention }\end{array}$} & \multicolumn{3}{|c|}{$\begin{array}{l}\text { Difference }(\mathrm{N}=67) \\
\text { Exercise versus control group }\end{array}$} & \multirow[t]{2}{*}{ P-value } \\
\hline & Mean & SD & Mean & SD & Mean & SE & $95 \% \mathrm{Cl}$ & \\
\hline Weight $(\mathrm{kg})$ & -0.6 & 2,1 & -0.8 & 2,9 & 0.3 & 0.6 & $-1.0-1.5$ & 0.68 \\
\hline Body mass index $\left(\mathrm{kg} / \mathrm{m}^{2}\right)$ & -0.3 & 0.7 & -0.4 & 1.1 & 0.1 & 0.2 & $-0.3-0.6$ & 0.55 \\
\hline Fat $(\%)$ & 9.3 & 15.2 & 5.9 & 11.7 & 2.9 & 3.3 & $-3.6-9.4$ & 0.37 \\
\hline $\mathrm{VO}_{2 \max }(\mathrm{I} / \mathrm{min})$ & 0.3 & 0.4 & -0.0 & 0.4 & 0.4 & 0.1 & $0.2-0.5$ & $0.000^{\mathrm{a}}$ \\
\hline HR at steady state (bpm) & -10 & 11 & 0 & 10 & -10 & 3 & $-15.2--4.6$ & $0.000^{\mathrm{a}}$ \\
\hline $\begin{array}{l}\text { Relative } \mathrm{VO}_{2 \max }(\mathrm{ml} / \mathrm{min} / \mathrm{kg}) \\
\text { Isometric muscle strength }(\mathrm{nm})\end{array}$ & 3.9 & 4.4 & 0.3 & 4.5 & 3.7 & 1.1 & $1.5-5.9$ & $0.001^{a}$ \\
\hline Shoulder (dominant) & 3.9 & 2.7 & 7.1 & 34.9 & -3.5 & 7.6 & $-18.6-11.6$ & 0.65 \\
\hline Arms (dominant) & 0.7 & 14.8 & -1.0 & 16.1 & 2.1 & 3.6 & $-5.1-9.4$ & 0.56 \\
\hline Abdomen & -7.1 & 29.0 & -7.7 & 30.2 & 0.7 & 7.0 & $-13.3-14.7$ & 0.92 \\
\hline Back & -17.7 & 49.1 & -16.2 & 32.7 & 1.7 & 8.3 & $-14.9-18.4$ & 0.84 \\
\hline Leg (right) & -2.0 & 54.0 & -5.7 & 37.8 & 2.7 & 9.7 & $-16.6-22.1$ & 0.77 \\
\hline Leg (left) & -4.1 & 20.2 & -7.4 & 28.6 & 0.7 & 4.7 & $-8.6-10.1$ & 0.88 \\
\hline Hand grip (dominant) & -0.4 & 5.5 & -0.5 & 5.5 & -0.1 & 1.2 & $-2.6-2.3$ & 0.90 \\
\hline Systolic BP (mm Hg) & -1.8 & 13.4 & -2.0 & 11.3 & 0.8 & 2.9 & $-4.9-6.6$ & 0.77 \\
\hline Diastolic BP (mm Hg) & -1.9 & 10.4 & -2.9 & 11.2 & 1.6 & 2.4 & $-3.2-6.4$ & 0.51 \\
\hline Total cholesterol (mmol/l) & -0.2 & 0.7 & 0.1 & 0.6 & 0.2 & 0.7 & $-0.1-0.4$ & 0.56 \\
\hline HDL cholesterol(mmol/l) & 0.0 & 0.1 & 0.0 & 0.2 & 0.0 & 0.0 & $-0.1-0.1$ & 0.78 \\
\hline LDL cholesterol (mmol/I) & 0.0 & 0.4 & 0.0 & 0.5 & 0.0 & 0.1 & $-0.2-0.3$ & 0.77 \\
\hline Triglyceride $(\mathrm{mmol} / \mathrm{l})$ & 0.0 & 0.7 & 0.0 & 0.6 & -0.0 & 0.1 & $-0.3-0.3$ & 0.80 \\
\hline
\end{tabular}

a Significant change $(\mathrm{P}<0.05)$. 


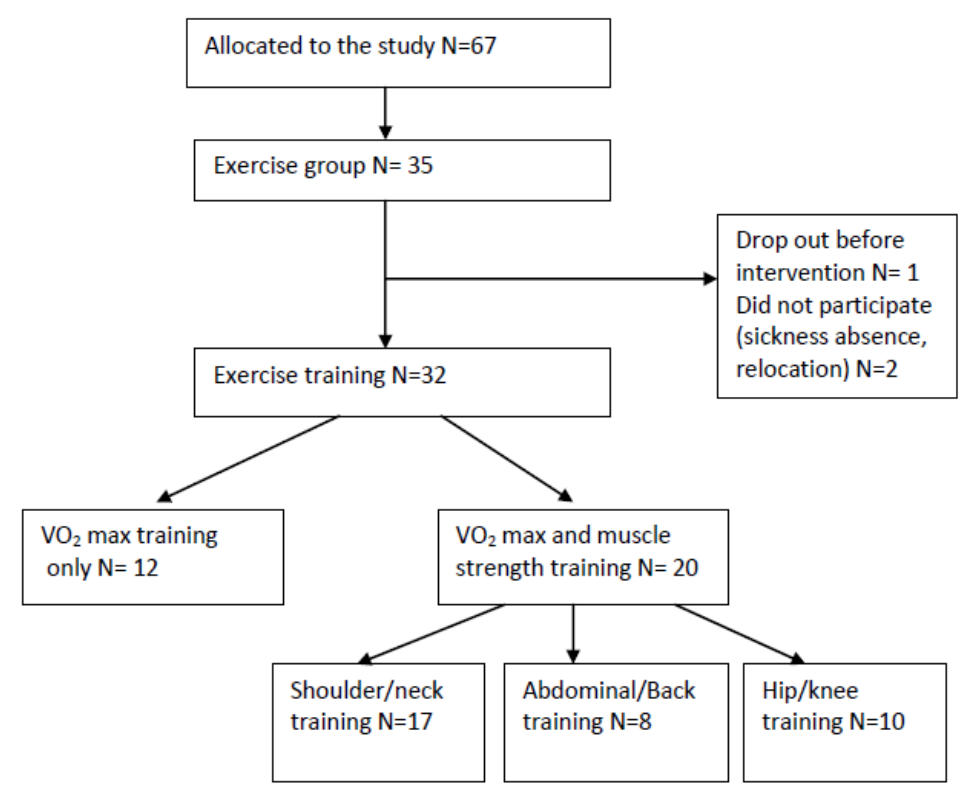

Figure 2. Allocation in individual muscle strength training groups.

\section{Discussion}

The major finding in this study was a significantly increased $\mathrm{VO}_{2 \max }$ among the construction workers following 20 minutes of aerobic capacity training (minimum $70 \%$ of $\left.\mathrm{VO}_{2 \max }\right) 3$ times a week for 3 months. The $12.5 \%$ increase in $\mathrm{VO}_{2 \max }$ is likely to provide clinically relevant decreased risk for metabolic and cardiovascular disorders. Blair et al (19) have shown that risk of allcause mortality significantly decreases when relative $\mathrm{VO}_{2 \max }$ is $>30 \mathrm{ml} / \mathrm{min} / \mathrm{kg}$. In our study, the mean relative $\mathrm{VO}_{2 \max }$ increased from 27.1 (SD 6.9) to 31.0 (SD 7.3) $\mathrm{ml} / \mathrm{min} / \mathrm{kg}$ in the exercise group. A high percentage of the construction workers in this study were observed to have a low $\mathrm{VO}_{2 \max }$ at baseline with none of the participants at baseline having a high relative $\mathrm{VO}_{2 \max }$ and only 7 out of 67 participants were classified as having moderate relative $\mathrm{VO}_{2 \max }$. Because workers with high physical work demands and low $\mathrm{VO}_{2 \max }$ are shown to have an excessive risk for cardiovascular mortality (3), the findings of low $\mathrm{VO}_{2 \max }$ of the construction workers in our study may indicate that they have a increased risk for developing cardiovascular disease. Thus the finding of a clinically relevant increase in $\mathrm{VO}_{2 \max }$ among construction workers in response to a relatively low dose of exercise in this study is promising for reducing general excessive risk for cardiovascular disease among construction workers. Particularly the youngest group, who had a general low fitness level, seemed to have highly benefitted from $\mathrm{VO}_{2 \max }$ training, which may call for health-related initiatives particularly targeting this group. However, the intervention could not demonstrate significant results of the muscle training at
$60 \%$ of 1 RM on strength measured as MVC. Actually, the relatively low intensity of the muscle strength training program was chosen after careful consideration of how to reduce the potential risk of pain and overload, but may mean that we could not expect an increase in strength. Nevertheless, this does not exclude a contribution to the effect on aerobic capacity. In this study, the focus was on tailoring a specific health-related lifestyle intervention to a specific target group. Based on health check results, each worker was allocated to an individual exercise program in a standardized protocol. However, when individual training programs are made within the intervention group, this increases the heterogeneity and therefore reduces power and the possibility to demonstrate statistically significant general effects in muscle strength. This highlights the challenge of a study design focused on individual interventions targeted to specific groups defined by, for example, work exposure, work demand, and individual health profiles. On the other hand, the protocol for distribution of the individual exercise groups in this study may be a model for designing similar studies in the future.

Other studies have shown positive effects of training interventions on muscle strength in occupational settings, but, in contrast to our study, the study groups were bigger and training protocols similar for all participants $(20,21)$.

In spite of a relatively high attendance rate, 15 of 34 participants reported that lack of time was a reason for absence from the training session. The sessions took place during working hours with the approval of employers and at the workplace for most of the participants. But despite this, and even with supervised train- 
ing by professionals, it was still a challenge to motivate people to adhere to the training program.

The limitation of the study is the low acceptance within this job group to participate in exercise training. We recruited eligible participants well above the required number according to our power calculation. However, there was a high decline rate $(\sim 1 / 3)$ among those who replied to our invitation $(\sim 2 / 3)$ resulting in group sizes below those estimated in the power calculation.

In conclusion, this study demonstrated 12 weeks of short exercise bouts integrated in the workday of constructions workers to increase $\mathrm{VO}_{2 \max }$ significantly has a clinically relevant positive effect size regarding risk factors for cardiometabolic disorders. This underlines the effectiveness of exercise training in workplace settings.

\section{Acknowledgments}

The authors would like to thank biomedical laboratory technician Kirsten Kjaer, physiotherapist Klaus Hansen, and laboratory technician Dorte Ekner for their support with tests and measurements. Furthermore, thanks to all exercise instructors, physiotherapy director Marianne Thomsen, and the research staff at the department of Endocrinology at Hospital of Southwest Denmark, Esbjerg. The FINALE project study was supported by a grant from the Danish Working Environment Research Foundation and the Ministry of Culture Committee on Sports Research, Denmark. The authors declare no conflict of interest.

\section{References}

1. Fang J, Wylie-Rosett J, Cohen HW, Kaplan RC, Alderman MH. Exercise, body mass index, caloric intake, and cardiovascular mortality. Am J Prev Med. 2003 Nov;25(4):283-9. http:// dx.doi.org/10.1016/S0749-3797(03)00207-1 .

2. Haapanen N, Miilunpalo S, Vuori I, Oja P, Pasanen M. Characteristics of leisure time Physical activity associated with decreased risk of premature all-cause and cardiovascular disease mortality in middle-aged men. Am J Epidemiol. 1996 May 1;143(9):870-80.

3. Holtermann A, Mortensen OS, Burr H, Sogaard K, Gyntelberg F, Suadicani P. Physical demands at work, physical fitness, and 30 -year ischaemic heart disease and all-cause mortality in the Copenhagen Male Study. Scand J Work Environ Health. 2010 Sep;36(5):357-65.

4. Holtermann A, Jorgensen MB, Gram B, Christensen JR, Faber A, Overgaard K, et al. Worksite interventions for preventing physical deterioration among employees in jobgroups with high physical work demands: background, design and conceptual model of FINALE. BMC Public Health. 2010;10:120. http://dx.doi.org/10.1186/1471-2458-10-120.

5. Paffenbarger RS, Hyde R, Wing AL, Hsieh Cc. Physical activity, all-cause mortality, and longevity of college alumni. N Engl J of Med. 1986 Mar 6;314(10):605-13. http://dx.doi. org/10.1056/NEJM198603063141003.

6. Sofi F, Capalbo A, Cesari F, Abbate R, Gensini GF. Physical activity during leisure time and primary prevention of coronary heart disease: an updated meta-analysis of cohort studies. Eur J Cardiovasc Prev Rehabil. 2008 Jun;15(3):247-57. http:// dx.doi.org/10.1097/HJR.0b013e3282f232ac.

7. Krause N, Brand RJ, Kaplan GA, Kauhanen J, Malla S, Tuomainen TP, et al. Occupational physical activity, energy expenditure and 11-year progression of carotid atherosclerosis. Scand J Work Environ Health. 2007 Dec;33(6):405-24.

8. Schneider S, Becker S. Prevalence of physical activity among the working population and correlation with work-related factors: results from the first German National Health Survey. J Occup Health. 2005 Sep;47(5):414-23. http://dx.doi. org/10.1539/joh.47.414.

9. Hoonakker P, van Duivenbooden C. Monitoring working conditions and health of older workers in Dutch construction industry. Am J Ind Med. 2010;53(6):641-53. http://dx.doi. org/10.1002/ajim.20838.

10. Groeneveld I, Proper K, van der Beek A, van Duivenbooden C, van Mechelen W. Design of a RCT evaluating the (cost-) effectiveness of a lifestyle intervention for male construction workers at risk for cardiovascular disease: The Health under Construction study. BMC Public Health. 2008;8(1):1-12. http://dx.doi.org/10.1186/1471-2458-8-1.

11. Arndt V, Rothenbacher D, Daniel U, Zschenderlein B, Schuberth S, Brenner H. Construction work and risk of occupational disability: a ten year follow up of 14474 male workers. Occup Environ Med. 2005 Aug 1;62(8):559-66. http://dx.doi.org/10.1136/oem.2004.018135.

12. Holmstrom E, Moritz U, Engholm G. Musculoskeletal disorders in construction workers. Occup Med. 1995 Apr;10(2):295-312.

13. Astrand PO, Ryhming I. A nomogram for calculation of aerobic capacity (physical fitness)from pulse rate during submaximal work. J Appl Physiol. 1954;7(2):218-21.

14. Faber A, Hansen K, Christensen H. Muscle strength and aerobic capacity in a representative sample of employees with and without repetitive monotonous work. Int Arch Occup Environ Health. 2006 Jan 1;79(1):33-41. http://dx.doi. org/10.1007/s00420-005-0025-z.

15. Strand I. Aerobic work capacity in men and women with special reference to age. Acta Physiol Scand. Suppl. 1960;49(169):1-92.

16. Essendrop M, Schibye B, Hansen K. Reliability of isometric muscle strength tests for the trunk, hands and shoulders. Int J Ind Ergon. 2001 Dec;28(6):379-87. http://dx.doi. org/10.1016/S0169-8141(01)00044-0.

17. Andersen UO, Henriksen JH, Jensen G. Sources of measurement variation in blood pressure in large-scale epidemiological 
surveys with follow-up. Blood Press. 2002;11(6):357-65. http://dx.doi.org/10.1080/080370502321095320.

18. Berg CM, Lissner L, Aires N, Lappas G, Toren K, Wilhelmsen L, et al. Trends in blood lipid levels, blood pressure, alcohol and smoking habits from 1985 to 2002: results from INTERGENE and GOT-MONICA. Eur J Cardiovasc Prev Rehabil. 2005 Apr;12(2):115-25.

19. Blair SN, Kohl HW, III, Paffenbarger RS, Jr., Clark DG, Cooper KH, Gibbons LW. Physical fitness and all-cause mortality. A prospective study of healthy men and women. JAMA. 1989 Nov 3;262(17):2395-401. http://dx.doi. org/10.1001/jama.262.17.2395.

20. Pedersen MT, Blangsted AK, Andersen LL, Jorgensen MB, Hansen EA, Sjogaard G. The effect of worksite physical activity intervention on physical capacity, health, and productivity: a 1-year randomized controlled trial. J Occup Environ Med. 2009 Jul;51(7):759-70. http://dx.doi. org/10.1097/JOM.0b013e3181a8663a.

21. Andersen LL, Saervoll CA, Mortensen OS, Poulsen OM, Hannerz H, Zebis MK. Effectiveness of small daily amounts of progressive resistance training for frequent neck/shoulder pain: Randomised controlled trial. Pain. 2011 Feb;152(2):440-6. http://dx.doi.org/10.1016/j.pain.2010.11.016.

22. Shvartz E, Reibold RC. Aerobic fitness norms for males and females aged 6 to 75years: a review. Aviat Space Environ Med. 1990;61:3-11.

Received for publication: 11 August 2011 